\title{
Web-Enabled Spatial Decision Analysis Using Ordered Weighted Averaging (OWA)
}

\section{Claus Rinner}

University of Münster

Jacek Malczewski

University of Western Ontario

\section{Please Cite:}

Rinner, C., \& Malczewski, J. (2002). Web-enabled spatial decision analysis using ordered weighted averaging (OWA). Journal of Geographical Systems, 4(4), 385-403.

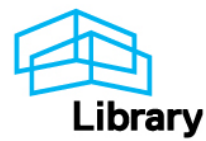




\title{
Web-enabled spatial decision analysis using Ordered Weighted Averaging (OWA)
}

\author{
Claus Rinner ${ }^{1}$, Jacek Malczewski ${ }^{2}$ \\ ${ }^{1}$ Institute for Geoinformatics (IfGI), University of Münster, Robert-Koch-Str. 26-28, 48149 \\ Münster, Germany (e-mail: rinner@ifgi.uni-muenster.de) \\ ${ }^{2}$ Department of Geography, University of Western Ontario, London, Ontario N6A 5C2, \\ Canada (e-mail: jmalczew@uwo.ca)
}

Received: 24 September 2002 / Accepted: 10 January 2003

\begin{abstract}
This paper presents a spatial decision support tool that implements the Ordered Weighted Averaging (OWA) method. OWA is a family of multicriteria evaluation operators characterised by two sets of weights: criterion importance weights and order weights. We propose a highly interactive way of choosing, modifying, and fine-tuning the decision strategy defined by the order weights. This exploratory approach to OWA is supported by a graphical representation of the operator's behaviour in terms of decision risk and tradeoff/dispersion between criteria. Our prototype implementation is based on the CommonGIS software, and thus, Webenabled and working with vector data. We successfully demonstrate online, exploratory support of spatial decision strategies using a data set of skiing resorts in Wallis, Switzerland.
\end{abstract}

Key words: Web-enabled spatial decision analysis, multicriteria evaluation, Ordered Weighted Averaging (OWA), Geographic Information Systems

JEL classification: C61, D89, R29, Z00

\section{Introduction}

In the most general terms, multicriteria decision problems involve a set of decision alternatives that are evaluated on the basis of conflicting and incommensurate criteria. Central to GIS-based multicriteria analysis is the integration of geographical data (map layers) and judgments (preferences and uncertainty) to provide an overall assessment of the decision alternatives. This is accomplished by an appropriate multicriteria decision rule or

\footnotetext{
We gratefully acknowledge support from Gennady and Natalia Andrienko, and Hans Voß for providing access to parts of the CommonGIS source code and to a preliminary version of the developer's manual. Piotr Jankowski provided helpful suggestions on improving the manuscript and supported a leave of absence for cooperation between the authors. We also thank two anonymous reviewers for their comments on an earlier draft of this manuscript.
} 
evaluation function. It is the decision rules that dictate how to rank alternatives or to decide which alternative is preferred over another.

Over the last decade or so, a number of multicriteria evaluation methods have been implemented in the GIS environment including weighted linear combination (WLC) and its variants (Janssen and Rietveld 1990; Eastman 1997), ideal point methods (Carver 1991; Jankowski 1995; Pereira and Duckstein 1996), concordance analysis (Carver 1991; Joerin et al. 2001), and analytical hierarchy process (Banai 1993; Eastman 1997). Among these procedures, WLC and Boolean overlay operations, such as intersection (AND) and union (OR), are considered the most straightforward and the most often employed (Malczewski 1999). These operations have been generalized using the Ordered Weighted Averaging (OWA) concept developed by Yager (1988). One of the major characteristics of OWA is that it allows decision-makers to change the form of attribute (criterion) combinations from a minimum-type (logical AND) combination through all intermediate types (including WLC) to a maximum-type (logical OR) combination (Yager 1988; Jiang and Eastman 2000). This property provides a sound basis for designing a Web-enabled decision analysis tool with the capacity of exploring different decision rules.

In the recent years much research effort has been focused on integrating GIS capabilities and multicriteria evaluation methods in the context of Webbased decision support tools (for an overview see Carver 1999). The simplest and most popular geographic information-enabled Internet applications are route planning and real-estate applications. For example, services at MapQuest (2002) including driving directions and a road trip planner can be considered as simple decision support tools. The Canadian Real Estate Association provides another example of Web-based spatial decision support. The association maintains a gateway to provincial real estate listings (MLS 2002). The search for a property by its very nature is a spatial multicriteria decision problem. Accordingly, the users of the MLS gateway create a database query with their search criteria providing them with a ranking of real estate properties. This ranking can be interpreted as a suggestion for a choice of properties for further inspection.

There are several more advanced Web-based spatial multicriteria applications (Carver et al. 1996; Menegolo and Peckham 1996; Barghava and Tettelbach 1997; Wan et al. 1999; Zhu and Dale 2001; Zhu et al. 2001). For example, Carver et al. (1996) describe Open Spatial Decision Making (OSDM). Users of OSDM can switch constraints on or off, and weight factors. The underlying GIS calculates suitable locations according to the users' input and the WLC decision rule. A map image is presented with a continuous colour scale, ranging from excluded to qualified areas. Users can interact with the map to locate their preferred site. This information along with an indication of how confident one is about the choice can be sent to the contact persons.

Menegolo and Peckham (1996) propose multicriteria evaluation techniques integrated with GIS (MC-GIS). The authors use an ideal point method and concordance analysis for a site selection problem. A case study providing a viewer for GIS data, forms for data selection and user preference input, and a map display for evaluation results, used to be accessible on the Internet. Malczewski (1999) examines MC-GIS in more detail. 
Barghava and Tettelbach (1997) describe a Web-based decision support prototype for recycling waste disposal. The system helps users to find the optimal route for dispensing a collection of waste items at recycling stations. An optimisation tool is used in conjunction with HTML forms to calculate a route with best tradeoff between revenue/charge for disposal and travel time between visited stations.

Wan et al. (1999) and Zhu et al. (2001) present a Web-based system that uses the analytical hierarchy process (AHP) as multicriteria decision rule. Zhu et al. (2001) propose a vegetation manager that provides access to decision support tools. The VegMan prototype includes JavaAHP, an online multicriteria evaluation component that implements the analytical hierarchy process. This tool helps users to assess management options in regional vegetation management on the base of information on facts and policies that is available in VegMan, combined with their own preferences (see also Zhu and Dale 2001). In addition to the use of the AHP decision rule, Wan et al. (1999) propose a Web-based system that has the capability of combining multiple decisionmakers' rankings of alternatives by combining the ideal point method and the Border's Choice rule in the GIS environment (see Malczewski 1996).

The ideal point decision rule is also used in a demo project of the CommonGIS software that provides decision support for the selection of a skiing resort in the Wallis region in Switzerland (Wallis 2002). CommonGIS helps users to explore spatially referenced data with highly interactive maps. It contains a map-centred implementation of the ideal point technique that was first realized in the DECADE prototype (Jankowski et al. 2001). The CommonGIS system is presented in Andrienko and Andrienko (1999) under its former name Descartes (see Sect. 3 for a more detailed description of the system).

To summarize, there is only a limited number of spatial multicriteria decision support applications available on the Web today. Many tools do not assist users in choosing between decision alternatives, but provide decision support by facilitating information access and visualization. Some of the applications do not describe the decision rules they use. In addition, most available systems are custom-built for specific applications or data. To our knowledge, there are no generic tools that would accept user-defined data online, and hence act as a Web-based multicriteria evaluation service in the sense of state-of-the-art application service providing (ASP).

The available systems also differ with respect to their capabilities to accommodate the user's preferences in terms of decision rule, decision criteria, and criterion weights. Accordingly, we suggest four classes of Webenabled spatial decision analysis tools characterized by their decision support capabilities:

(i) evaluation of a single, fixed criterion or optimisation function (e.g. driving distance in route planner, cost in recycling decision support system),

(ii) database queries with user-defined Boolean criteria such as AND and OR (e.g. real-estate listing),

(iii) use of fixed decision rule with user-defined criteria and importance weights (e.g. nuclear waste, skiing resorts), and

(iv) selection of decision rule (no application known). 
This paper offers a Web-enabled multicriteria decision analysis tool that allows users to select a decision rule, choose criteria and set criterion weights, addressing type (iv) in the above classification. It presents an implementation of the OWA decision rules in the CommonGIS system. In Sect. 2, we provide an overview of the OWA method. Section 3 discusses the implementation of the OWA concept in CommonGIS. The use of the Web-enabled system is then presented and illustrated using the skiing resort selection problem in the Wallis region, Switzerland. The final section presents conclusions and an outlook on further research directions emerging from our results.

\section{Ordered Weighted Averaging (OWA)}

OWA is a class of multicriteria operators developed by Yager (1988). OWA involves two vectors of weights: criterion importance weights $\left(w_{j}, j=1,2, \ldots\right.$, $n$ ) and order weights $\left(v_{j}\right)$. The importance weight $w_{j}$ is assigned to the $j$-th criterion (attribute) for all locations to indicate its relative importance (according to the decision-maker's preferences) in the set of criteria under consideration. The order weights are associated with the criterion values on a location-by-location (object-by-object) basis. They are assigned to a location's standardized attribute values in decreasing order without considering which attribute the value comes from. The reordering procedure is central to the OWA method. It involves associating an order weight $v_{j}$ with a particular ordered "position" of the weighted attribute values. The first order weight, $v_{1}$, is assigned to the highest weighted attribute values for each location, $v_{2}$ is associated with the second highest values, and so on; $v_{n}$ is assigned to the lowest values. Thus, a particular order weight $v_{j}$ is not associated with a particular attribute but rather with a particular ordered position of an attribute value $a_{i j}$.

Given a set of weighted attribute values $\left(w_{1} a_{i 1}, w_{2} a_{i 2}, \ldots, w_{n} a_{i n}\right)$ and a set of order weights $\left(v_{1}, v_{2}, \ldots, v_{n} ; 0 \leq v_{j} \leq 1\right.$, and $\left.\operatorname{sum}\left(v_{j}\right)=1\right)$, OWA can be defined as follows: OWA $=\operatorname{sum}\left(v_{j} b_{i j}\right)$, where $b_{i 1} \geq b_{i 2} \geq \ldots \geq b_{i n}$ is the sequence obtained by reordering the weighted attribute values, $w_{1} a_{i 1}, w_{2} a_{i 2}$, $\ldots, w_{n} a_{i n}$. Specifically, the computation of OWA involves three main steps:

(i) define order weights,

(ii) sort the weighted standardized criterion values of each alternative in descending order,

(iii) multiply values by corresponding order weights and sum up to build an evaluation score for a given location.

To illustrate OWA, let us consider a set of weighted attribute values associated with the $i$-th location, $w_{j} a_{i j}=(0.8,0.1,0.9,0.5)$ and a set of associated order weights $v_{j}=(0.4,0.3,0.2,0.1)$. Given the inputs, the OWA procedure involves: reordering the weighted attribute values as follows: $b_{i 1}=0.9, b_{i 2}=0.8, b_{i 3}=0.5$, and $b_{i 4}=0.1$, and combining the ordered weighted attribute values; that is, $O W A=(0.4 \times 0.9)$ $+(0.3 \times 0.8)+(0.2 \times 0.5)+(0.1 \times 0.1)=0.71$.

The generality of OWA is related to its capability to implement a wide range of combination operators by selecting an appropriate set of order weights (Yager 1988). Four of these operators are of particular importance (see Table 1). Assigning equal order weights (that is, $v_{j}=1 / n$ ) results in the 
Table 1. Selected OWA operators and associated order weights, and measures of the operator's combination behaviour

\begin{tabular}{lllll}
\hline OWA operator & Order weights & ORness & Tradeoff & Dispersion \\
\hline MIN (AND) & $v_{n}=1 ; v_{j}=0$, otherwise & 0.0 & 0.0 & 0.0 \\
WLC & $v_{j}=1 / n$, for all $j$ & 0.5 & 1.0 & 1.0 \\
MEDIAN & $\begin{array}{l}\text { For } n \text { odd: } v_{(n+1) / 2}=1 ; \\
\quad v_{j}=0, \text { otherwise }\end{array}$ & & & \\
& $\begin{array}{l}\text { For } n \text { even: } v_{n / 2}=v_{(n / 2)+1}=0.5 ; \\
v_{j}=0, \text { otherwise }\end{array}$ & 0.5 & 0.0 & 0.0 \\
MAX (OR) & $v_{1}=1 ; v_{j}=0$, otherwise & 1.0 & 0.0 & 0.0 \\
\hline
\end{tabular}

combination according to the weighted linear combination (WLC). Order statistics including the MEDIAN can be recovered by an appropriate specification of the order weights. The MIN and MAX operations represent the extreme cases of OWA and they correspond to the logical AND and OR operations, respectively. The position of OWA on the continuum between the extreme cases can be identified by specifying the degree of ORness (or ANDness) (Yager 1997). The measure of ORness is defined as: $\operatorname{Sum}\left(v_{j}(n-j) /\right.$ $(n-1))$. The measure ranges from 0 to 1 . It measures the degree to which an OWA operator is similar to the logical OR (or the MAX operator) in terms of its combination behaviour. Dual to ORness is the measure of ANDness; that is, ANDness $=1-$ ORness. Therefore, OWA is also referred to as an ORAND operator since it may act as a combination of both ORing and ANDing.

The measure of ORness can be interpreted in the context of wellestablished behavioural theory of decision-making (Bodily 1985; Yager 1988). According to the theory an essential component of any decisionmaking process is the attitude of the decision-maker (individual or organization) towards risk. Risk perception or risk propensity is defined as the consistency of a decision-maker to either take or avoid actions that he/she perceives as risky. There are both theoretical and empirical evidence to show that an individual with low risk-taking propensity will typically weigh negative outcomes more highly and, conversely, an individual with high risk-taking propensity is more likely to weigh positive outcomes more highly (Bodily 1985). Accordingly, ORness can be recognized as a measure of the degree of the decision-maker's optimism (Yager 1988). The values of ORness greater than 0.5 represent optimistic decision strategies, while the values less than 0.5 represent pessimistic strategies. If ORness $=0.5$, then a decision-maker is indifferent towards risk, or risk neutral. Thus, OWA can accommodate varying degrees of optimism of decision-makers.

In addition to ORness, OWA operators can be characterized by the measures of tradeoff and dispersion (Yager 1988; Jiang and Eastman 2000). The former is calculated as follows: tradeoff $=1-\operatorname{Sqrt}\left(n /(n-1) * \operatorname{Sum}\left(\left(v_{j}-1 /\right.\right.\right.$ $\left.n)^{2}\right)$ ). It is basically an inverse distance of order weights from equally distributed weights $(1 / \mathrm{n})$. Tradeoff is a measure for the substitutability (or compensation) of low values on one criterion by high values on another criterion.

Dispersion is similar to tradeoff in that it measures the "entropy" of order weights (Yager 1988). The formula is $(1 / \ln (n)) * \operatorname{Sum}\left(v_{j} * \ln \left(v_{j}\right)\right)$; it is a 
normalized sum of products of each order weight with its natural logarithm. The dispersion can be interpreted as a measure of utilization, by the OWA operators, of the information contained in the criteria. The more dispersed the order weights, the more information contained in the criteria is being used in the process of combining the criterion outcomes. This point can be demonstrated by comparing the dispersion of the order weights associated with the MEDIAN and WLC combinations. These combinations are characterized by the same ORness value of 0.5 . However, MEDIAN only uses the information of a single attribute associated with a given location, while WLC involves all attribute values. Consequently, the dispersion and tradeoff measures for MEDIAN are equal to 0.0 , while WLC is characterized by the maximum dispersion and tradeoff of 1.0 .

\section{Exploratory OWA in CommonGIS}

\subsection{The CommonGIS project and system}

"Common Access to Geographically Referenced Data" (CommonGIS) was a European Union research project aiming at creating easy-to-use visualizations of statistical and environmental data on thematic maps. Several demonstrator applications have been put online at the project's Web site (CommonGIS 2002a). The applications are based on the Descartes software that has been developed at the Fraunhofer Institute for Autonomous Intelligent Systems, Sankt Augustin, Germany (Fraunhofer 2002). Andrienko and Andrienko (1999) describe their system focusing on its interactivity to support visual exploration of geographic data. Among the interactive features are extensive manipulation options for the cartographic presentation of data, including maps linked with graph displays. Voß et al. (2000) describe the Portuguese Census Data demonstrator.

The CommonGIS software is implemented in the Java programming language and thus Internet-compliant as well as capable of running locally on a single computer. Starting the system in a Web browser means to download a Java applet that contains all the features of the system. A current version of the CommonGIS software has been published at CommonGIS (2002b) under a license that disallows any commercial use. The source code is only available to the extent that is needed to add new methods to the system. This proved to be a limitation and additional source code was provided to the authors so that the user interface appearance of the OWA implementation discussed in this paper could be streamlined with the existing code.

\subsection{Decision support in CommonGIS}

Jankowski et al. (2001) present a prototype software called DECADE that supports the exploration and structuring of spatial multicriteria decision problems. Interactive maps are used as a "visual index" for ordering decision alternatives and assigning criterion priorities. The authors implement mapbased multicriteria evaluation with the ideal point method. The proposed map-centred decision support techniques are demonstrated by means of a decision problem of primary health care funding in the State of Idaho. 
The ideal point method of DECADE has been inherited in the CommonGIS system as described in Sect. 1 for the Wallis demo application. It is reviewed here in more detail as it provides technical guidelines for implementing OWA as a second multicriteria evaluation method in CommonGIS. The conceptual approach to decision support in the feature-based CommonGIS system is that of calculating integrated evaluation scores in a table. The calculation uses tabular attributes of spatial alternatives represented by geographic features. Evaluation scores have to be stored in the feature table in order to become available for visualization. The ranking that is derived from evaluation scores is stored in that table as well. As a result, decision support in CommonGIS provides users with highly interactive tools for display of input data and evaluation results, and for interactive redefinition of the decision rule by manipulating criterion weights.

\subsection{Processing steps for decision support}

The flowchart in Fig. 1 outlines user interaction with the OWA tool, and processing of user selections and settings by the system. The boxes in the left and centre columns (light grey shading) represent steps that are shared with other decision support tools. The boxes in the right column of Fig. 1 (dark grey) represent steps that are specific to OWA.

Users have to select a table first. By doing so, the set of decision alternatives is defined to be the geographical features contained in the map layer the table belongs to. Next, the user will select a subset of the available attributes in the selected table to be used as decision criteria. The outcomes of each alternative with respect to each criterion have to be standardized by the system. The standardization method used in CommonGIS is score range transformation, i.e. for a benefit criterion, the minimum value is set to 0.0 , the maximum is set to 1.0, and all values in between are linearly transformed. For a cost criterion, scores are inverted. Now that criterion outcomes are made comparable, the user has to define the relative importance of criteria. Criterion weights take values between 0.0 and 1.0, and sum up to 1.0. The system multiplies criterion outcomes by the appropriate criterion weight to calculate weighted (standardized) criterion outcomes. Thereafter, users have to specify a decision strategy within the OWA family and/or define order

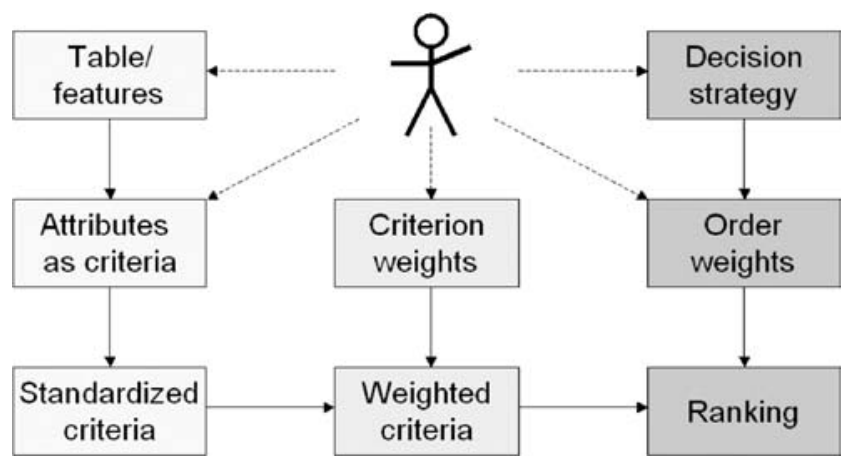

Fig. 1. User interaction, and processing of user selections and settings for ordered weighted averaging 
weights. Finally, order weights are multiplied with the ordered weighted criterion outcomes to calculate a score for each alternative (see Sect. 2). Scores are translated into a ranking that is displayed on a map. For the OWA-specific processing steps, custom user interface components were developed that are described in detail in the sequel.

\subsection{User interface for $O W A$}

The user interface of the OWA method in CommonGIS is characterized by its main functions:

- display of current order weights and OWA measures as indicators for selected decision strategy, and

- interactive definition and exploratory modification of decision strategy by

- choosing among default strategies, or

- modifying order weights, or

- modifying OWA measures.

The window of the OWA method has two tab folders, "Order weights" and "Criterion weights". The latter is on top when the window appears, and contains almost the same tools as the ideal point analysis window in the CommonGIS system. This tab is used to set types of criteria (benefit or cost) and relative importance weights for criteria.

The OWA-specific tools are contained in the "Order weights" tab shown in Fig. 2. To the left is a set of sliders and buttons to define the order weights. To the right is a graphical and textual display of the behaviour of the OWA operator, which corresponds to the current set of order weights. Tool tips with short descriptions of functions are provided when the mouse is pointed to any of the interactive tools in the "Order weights" tab.

The sliders for order weights are used to define each weight on a scale from 0.0 to 1.0. When the window first appears, order weights are set to equal values of $1 / \mathrm{n}$ to calculate an initial ranking of alternatives.

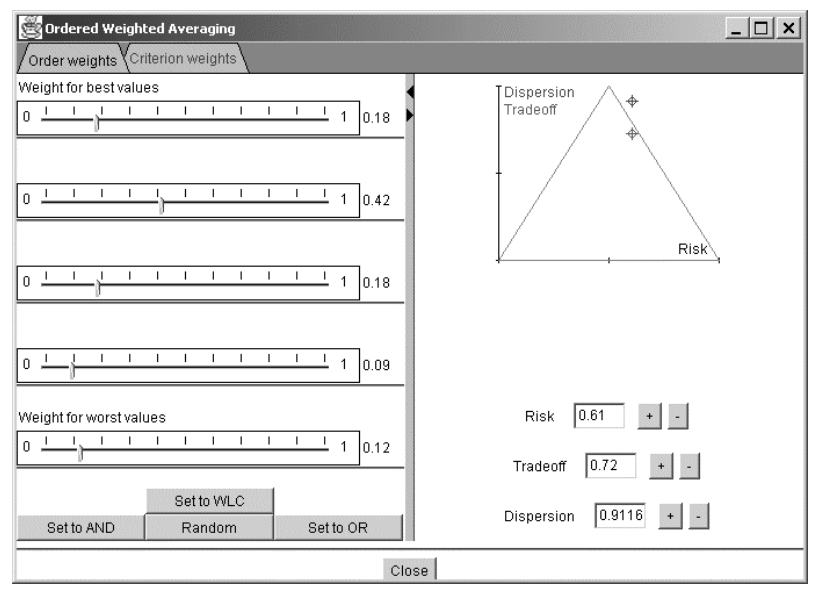

Fig. 2. Graphical user interface for OWA in CommonGIS 
Buttons are provided to quickly define order weights for three extreme cases of OWA. If the user clicks on the "Set to AND" button, the order weights are set to $(0,0, \ldots, 0,1)$. If the user clicks on the "Set to OR" button, the order weights are set to $(1,0, \ldots, 0,0)$. As mentioned in Section 2, AND is called a pessimistic approach, since alternatives are ranked according to their worst value. OR is called an optimistic strategy since bad values are accepted as long as an alternative has at least one outstanding property. Weighted linear combination (button "Set to WLC") uses equal order weights of $1 / n, n$ being the number of criteria considered. In this case, bad values in some criteria can be substituted by good values in others; alternatives score according to an average of their properties.

In addition, it is possible to get a random set of order weights. In this case, order weights $v_{1}, v_{2}, \ldots, v_{n}$ are chosen randomly under the following constraints: $0 \leq v_{j} \leq 1$, and $\operatorname{Sum}\left(v_{j}\right)=1$. This function can be used to test different sets of weights that range between the extremes described above. A random set of weights can also be used as a starting point for exploring different decision strategies manually, by manipulating the sliders for the order weights.

The right-hand frame of the OWA window displays measures of the behaviour of the current set of order weights. The behaviour is characterized by three measures: the degrees of risk (the ORness parameter), tradeoff and dispersion (see Sect. 2). The three corners of the triangle graph represent the three extreme cases in the OWA decision strategy space: AND, WLC, and OR (Jiang and Eastman 2000). The decision-maker can play with the "Random" button to examine the effects of different sets of order weights on the behaviour of the method.

Below the triangle, next to the text fields, which display the exact measures, are buttons to increase or decrease the respective measure. Risk/ORness is increased by increasing the order weight for the highest criterion outcome. Risk/ORness is decreased by decreasing the same weight. Tradeoff and dispersion are increased by decreasing the current maximal order weight (whichever it is), thus getting weights closer to equal distribution. Tradeoff and dispersion are decreased by increasing the current maximal weight. These adjustments are heuristic only. In most of the cases the effect cannot be inverted, and in some situations the measures are not changed in the desired direction. This is due to the fact that this implementation of the OWA method does not provide a means for mathematical deduction of order weights from given measures.

\subsection{Implementation of the method}

The OWA method has been integrated into CommonGIS as an additional decision support tool according to the programmer's manual that was made available by the developers. Additions and changes to the source code occurred exclusively in the package andr.analysis.calc (see Fig. 3).

The class CalcManagerImpl.java is a registry of available calculation methods. The new OWA method was listed in this registry by providing:

- an ID ("CalculateOWA"),

- a method name for display ("Ordered Weighted Averaging”),

- its main class name ("andr.analysis.calc.OWACalcDlg”). 


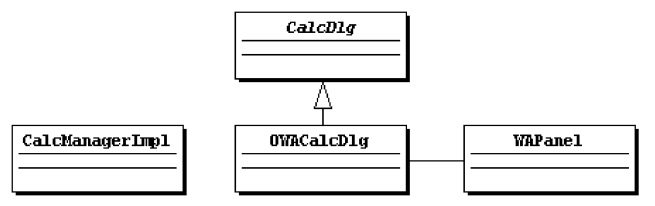

Fig. 3. Important classes in package andr.analysis.calc

Calculation classes are dynamically loaded into CommonGIS when required. Therefore, the presence or absence of the main class for a method decides about its availability to the user.

The main class for the OWA method is OWACalcDlg. It is a subclass of CalcDlg, which is a basic user interface for calculators. The Java interface Calculator requires the dialog classes to provide methods for showing their user interface, and for starting the calculation process. The OWA-specific user interface is displayed after inherited methods from class CalcDlg showed the dialogs to select a table, a calculation method, and attributes from the chosen table. The calculation is started with default values for order weights even before the OWA user interface initially appears, and later, whenever the user changes the order weights. Methods of CalcDlg also make a map appear on screen that shows the initial ranking of alternatives. By subclassing CalcDlg, OWACalcDlg inherits methods to connect to the table with attributes of alternatives and to the maps these geographic features are currently shown in. This way, calculated OWA scores can be stored, and updated, in the feature table, and visualization of OWA rankings is updated at every change of table values. The OWA user interface also contains an attribute weighting panel for selection of importance weights for decision criteria. This panel is realized through an association of an instance of WAPanel, a class in CommonGIS that was developed for the ideal point analysis method.

The code of the ideal point analysis method (IdealPointCalc.java) was used as a model for the OWA dialog. OWACalcDlg is a Frame; that is, a window in Java. Standard widgets from the Java 1.1 Abstract Window Toolkit (AWT) such as Button, TextField, and Canvas were used to build the user interface within the Frame. A custom CommonGIS TabbedPanel provides the layered tabs for criterion weights and order weights. A CommonGIS SplitLayout is used to arrange widgets in the adjustable left and right halves of the window.

More information about the OWA function can be found in the help menu under "decision support". The CommonGIS system help consists of HTML pages that are viewed with the operating system's standard Web browser. The existing decision support help page has been enlarged with information about how to use OWA. The help also describes the use of the "utility signs" cartographic method that supports understanding of OWA results (see following section).

\section{Sample application of Web-enabled OWA}

Many spatial decision support systems are limited to specific applications and specific data. The software presented here is designed as a generic decision support tool. The creation of specific applications is facilitated by: 
- the availability and openness of the software (download from Web site),

- installation instructions (online, and download from Web site), and

- method documentation (help page, and this paper).

In this paper we demonstrate OWA in CommonGIS using a specific decision problem. The demo application is available at OWA (2002).

The CommonGIS download package comes with a dataset about skiing resorts in the Wallis region in Switzerland. Forty skiing resorts are characterized by ten attributes that were acquired for a case study described in Voß et al. (2002). For the purpose of clarity of this paper, we use three out of the ten potential evaluation criteria. A hypothetical decision-maker plans a winter holiday trip and seeks a resort with enough lifts, an inexpensive ski pass for a week, and with non-expert level ski runs. This translates into the types of criteria and their relative importance as shown in Table 2. The number of ski lifts is to be maximized and gets a weight of 0.2 . The price for a ski pass and the percentage of expert level runs are to be minimized and get weights of 0.5 and 0.3 .

When describing our weighted OWA implementation, we will not deal with the problem of defining importance weights nor examine different sets of such weights (see Eastman 1997; Malczewski 1999; Thill 1999), but we will focus on the order weights that are central to the OWA approach (see Sect. 2).

Figure 4 shows the results of steps 1 to 3 of the OWA method as described above. The "utility signs" visualization method of CommonGIS (Andrienko and Andrienko 2002) is used to display standardized criterion outcomes for the skiing resorts in central Wallis.

Figure 5 shows a zoom to the five resorts in the "Vier Täler" region after importance weighting of criterion outcomes. The width of each bar is proportional to the importance weight of the corresponding criterion (cf. Table 2). Therefore, this cartographic method allows for a visual analysis of each alternative's suitability through the space occupied within the frame of the utility sign. The resulting ranks of alternatives after weighted linear combination (WLC) are, however, not obvious from visual inspection (column 4 of Table 3 ).

Figure 6 shows utility bars for the same area ordered by descending values. Utility is represented by the occupied surface, that is width $\times$ height, or in the attribute/criterion space, relative importance $\times$ standardized outcome. From Fig. 6 it follows that alternative 33 has a very good order-1 value and a good order-2 value, resulting in the best rank of these five alternatives. Alternative 35 has good order-1 and order-2 values, resulting in the second rank. Alternative 32 with a very good order-1 value, but bad order-2 and order-3 values receives only rank four.

We will now consider four decision strategies that will lead to four different decision rules within the family of OWA operators. Decision-maker 'A' seeks

Table 2. Decision criteria, types, and relative importance

\begin{tabular}{lll}
\hline Criterion & Criterion type & Importance weight \\
\hline Number of lifts & Benefit & 0.2 \\
Price for ski pass & Cost & 0.5 \\
Expert level runs & Cost & 0.3 \\
\hline
\end{tabular}




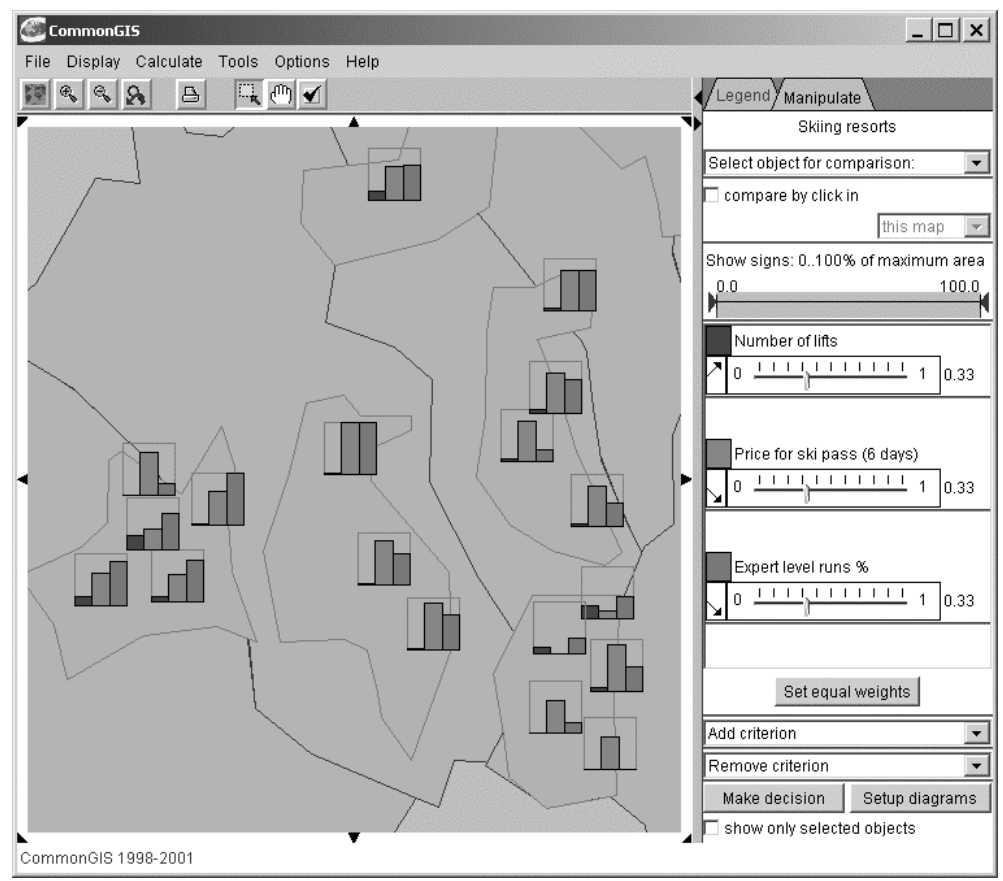

Fig. 4. Utility signs for standardized criterion outcomes

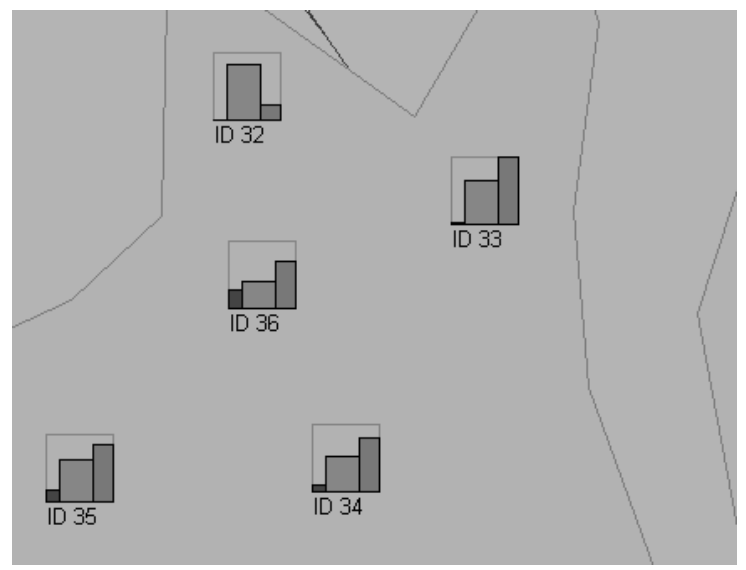

Fig. 5. Weighted standardized criterion outcomes for resorts in "Vier Täler" region

an alternative, which is outstanding in at least one criterion, its best criterion. The remaining criterion outcomes may be poor. ' $A$ ' takes the risk of considering only the best property of each skiing resort, irrespective of which criterion this actually is, and how bad the remaining criterion outcomes are. Visually, 'A' looks for the largest bar in first position in the ordered utility signs for each alternative. 
Table 3. Ranks of skiing resorts in the "Vier Täler" region (five resorts out of a total of 40 in Wallis) under different OWA decision strategies

\begin{tabular}{|c|c|c|c|c|c|c|}
\hline Operator & OR & OR-like & WLC & MEDIAN & AND-like & AND \\
\hline Scenario & 'A' & & 'B' & 'C' & & 'D' \\
\hline $\begin{array}{l}\text { Order } \\
\text { weights }\end{array}$ & $1.0,0.0,0.0$ & $0.5,0.3,0.2$ & $0.3,0.3,0.3$ & $0.0,1.0,0.0$ & $0.2,0.3,0.5$ & $0.0,0.0,1.0$ \\
\hline ID 32 & 7 & 26 & 29 & 36 & 32 & 37 \\
\hline ID 33 & 27 & 14 & 9 & 4 & 8 & 32 \\
\hline ID 34 & 34 & 30 & 26 & 8 & 22 & 10 \\
\hline ID 35 & 31 & 19 & 14 & 7 & 5 & 5 \\
\hline ID 36 & 37 & 33 & 30 & 16 & 23 & 2 \\
\hline
\end{tabular}

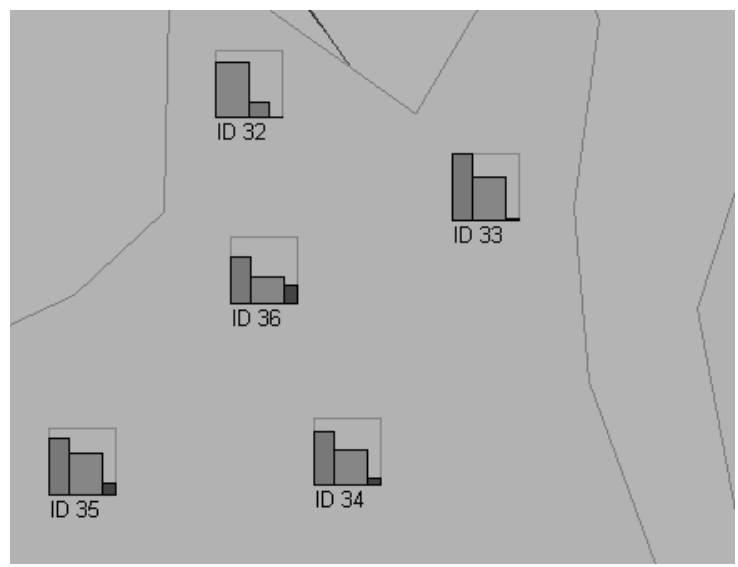

Fig. 6. Weighted standardized criterion outcomes ordered by descending utility values

The OWA method provides a means of defining this risk-taking decision strategy, which is equivalent to the logical OR operator. The corresponding vector of order weights $(1.0,0.0,0.0)$ gives full weight to the best criterion outcome for each alternative. Figure 7 shows the order weight definition in the OWA window, and the resulting measures of this optimistic strategy. Risk is maximal while tradeoff is minimal because full weight is given to a single criterion outcome. No compensation is possible between any two criteria (see Sect. 2). The result of the risk-taking OR strategy is shown in column 2 of Table 3 . Compared to WLC, alternative 32 with a single very good value improves its rank dramatically while all other alternatives deteriorate.

Decision-maker 'B' follows a decision strategy, which treats all criterion values as equal by applying order weights of 0.33 to each ordered value. The resulting ranks (column 4 of Table 3 ) are the same as with standard WLC (described above). Thus, WLC represents an intermediate level of decision risk and allows for full tradeoff between good and bad values.

In contrast, decision-maker ' $\mathrm{C}$ ' only allows for minimal compensation between values, but also accepts an intermediate risk level. 'C' gives full 


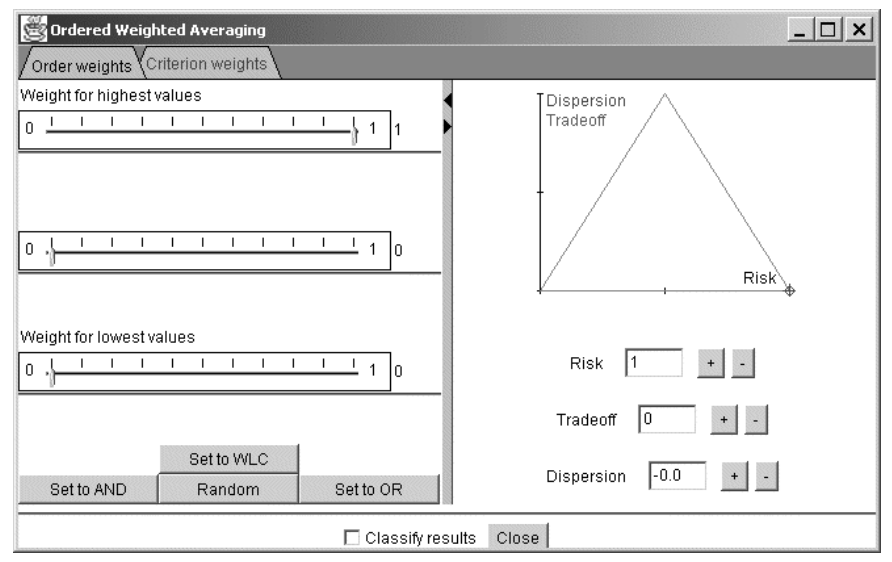

Fig. 7. Risk-taking decision strategy (OR operator within OWA family of operators)

emphasis to the criterion outcome in the middle position of ordered values, none to the best and worst position. This strategy corresponds to a MEDIAN operator. Alternatives 33 and 35 have relatively high values at the middle positions (cf. Fig. 6) and, thus, lead the ranking (column 5 of Table 3).

Finally, decision-maker ' $\mathrm{D}$ ' is cautious and prefers those decision alternatives that have fair outcomes even in their worst properties. An OWA strategy that considers only the worst criterion outcome for each alternative would be defined by order weights $(0.0,0.0,1.0)$. This strategy does not allow for compensation between good and bad values. Alternative 36 with the highest value in last position leads the ranking with this pessimistic strategy (column 7 in Table 3).

Table 3 summarizes the consequences of choosing different decision strategies in the Wallis location problem. Strategies are defined by vectors of order weights, and result in different rankings of decision alternatives. In addition to the four extreme strategies described so far, the table contains a moderately optimistic (OR-like, column 3), and a moderately pessimistic (AND-like, column 6) strategy, which might be more realistic than the extreme strategies. In the OR-like strategy, most weight is given to the best and intermediate outcomes. In the AND-like strategy, most weight is given to the worst and intermediate outcomes. Both strategies allow for some compensation between high and low criterion values.

Table 4 relates the ranking results to attribute space by summarizing the attribute values of the best alternatives (among the five observed) for each decision strategy. The consequences of attribute values on the outcomes of the OWA method are, however, not obvious from the values themselves, but are clearly revealed in the graphical display of ordered weighted criterion outcomes (see Fig. 6) as described in the sample scenarios.

We expected that the four decision strategies described in the above scenarios would result in four different rankings of alternatives. But are those rankings under two strategies statistically correlated? A visual analysis of correlations between rankings under any two of the described strategies is possible through an additional visualization option available in 
Table 4. Attribute values of top-ranked alternatives per decision strategy

\begin{tabular}{lccll}
\hline Strategy & ID & $\begin{array}{l}\text { Number of } \\
\text { lifts }\end{array}$ & $\begin{array}{l}\text { Price for } \\
\text { ski pass [SFR] }\end{array}$ & $\begin{array}{l}\text { Expert level } \\
\text { runs [\%] }\end{array}$ \\
\hline OR & 32 & 3 & 162.00 & 30.0 \\
OR-like & 33 & 4 & 215.00 & 0.0 \\
WLC & 33 & 4 & 215.00 & 0.0 \\
MEDIAN & 33 & 4 & 215.00 & 0.0 \\
AND-like & 35 & 26 & 222.00 & 5.71 \\
AND & 36 & 39 & 282.00 & 11.66 \\
\hline
\end{tabular}

CommonGIS. The scatter plots shown in Fig. 8 suggest that there is a linear correlation between WLC and MEDIAN strategies.

Statistical analysis with SPSS (see Table 5) confirmed a linear correlation between WLC and MEDIAN by a Spearman-Rho correlation coefficient of $0.781(\alpha<0.01)$. In addition, OR and WLC also have a significant correlation of $0.579(\alpha<0.01)$. Finally, the extreme OR and AND strategies have a significant negative correlation of $-0.425(\alpha<0.01)$.

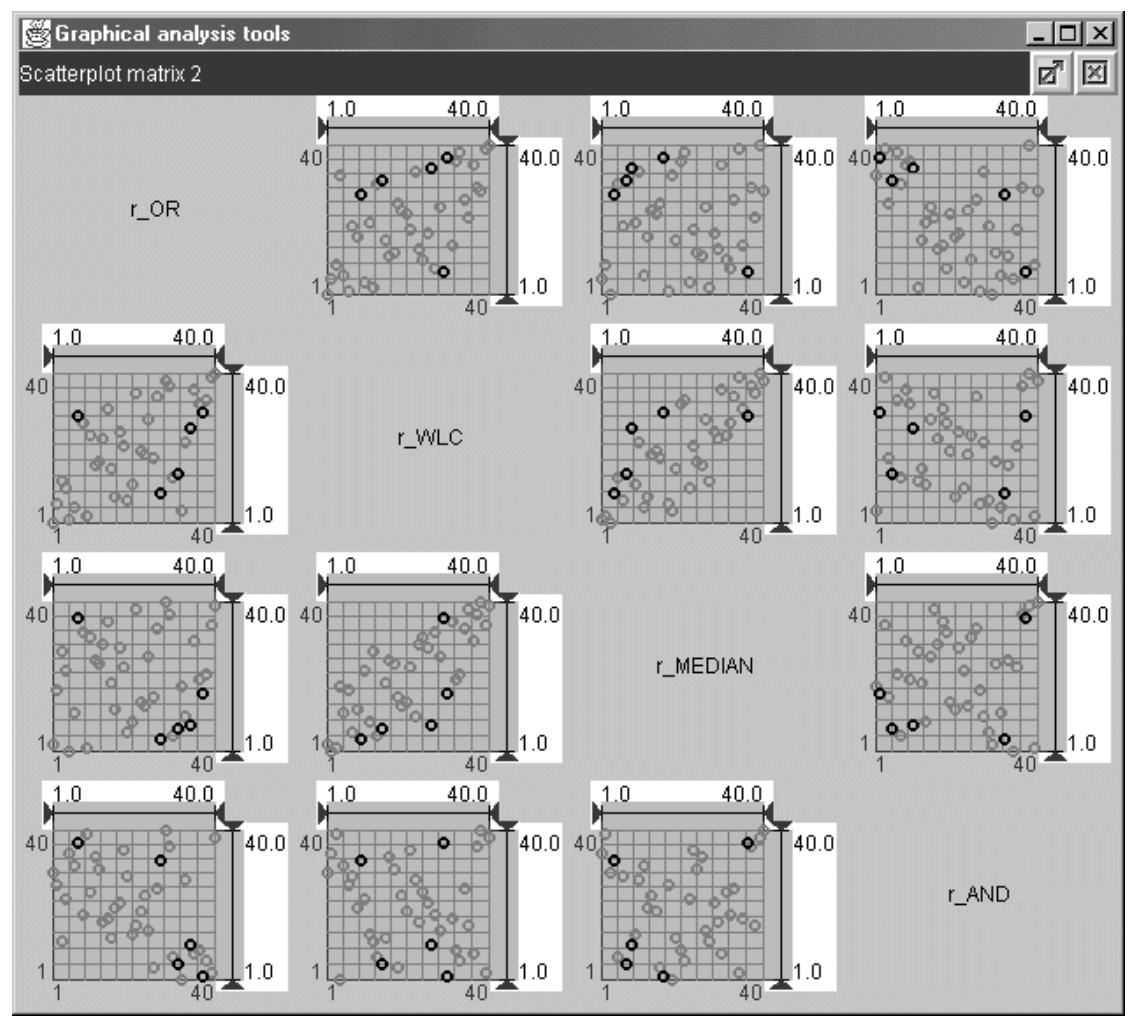

Fig. 8. Visual correlation between rankings under different decision strategies (dark circles represent observed resorts in the "Vier Täler" region) 
Table 5. Statistical correlation (Spearman-Rho) between rankings under different decision strategies

\begin{tabular}{|c|c|c|c|c|}
\hline & R_OR & R_WLC & R_MEDIAN & R_AND \\
\hline R_OR & 1.000 & .579 & .103 & -.425 \\
\hline $\begin{array}{l}\text { Corr. Coeff. } \\
\text { Sig. (2-tailed) }\end{array}$ & & .000 & .528 & .006 \\
\hline \multicolumn{5}{|l|}{ R WLC } \\
\hline Corr. Coeff. & .579 & 1.000 & .781 & -.120 \\
\hline Sig. (2-tailed) & .000 & & .000 & .461 \\
\hline \multicolumn{5}{|l|}{ R_MEDIAN } \\
\hline Corr. Coeff. & .103 & .781 & 1.000 & .075 \\
\hline Sig. (2-tailed) & .528 & .000 & & .646 \\
\hline \multicolumn{5}{|l|}{ R_AND } \\
\hline Corr. Coeff. & -.425 & -.120 & .075 & 1.000 \\
\hline Sig. (2-tailed) & .006 & .461 & .646 & \\
\hline
\end{tabular}

Comparisons made between decision strategies are affected by a number of factors that have been fixed in this sample application of OWA:

- the raw data at hand (data of 40 skiing resorts for the Wallis region),

- the selected criteria (three selected feature attributes, benefit or cost type, see Table 2),

- the standardization method (score range transformation),

- the criterion importance weights (see Table 2).

Thus, observations about correlation between the OWA results under different strategies cannot be generalized from one decision problem to another.

\section{Conclusion and outlook}

The Web-enabled Ordered Weighted Averaging (OWA) tool described in this paper provides access to a flexible spatial decision support technique for a broad range of potential users. OWA is a complex evaluation procedure that can be interpreted in a simplified manner in terms of decision risk and tradeoff between criteria. We attempted to demonstrate how these two dimensions can help to provide an easy-to-use interface to OWA that does not require full understanding of the mathematical operations involved. The graphical presentation of OWA methods in the strategy triangle provides a user-friendly visual control of the position of the chosen strategy relative to the extreme OWA operators. The usability of the prototype and its contribution to complexity reduction in spatial decision analysis particularly meet the requirements for Web-enabled applications.

The exploratory approach to spatial decision-making, which is founded on the base software, CommonGIS, helped us design a tool with built-in sensitivity analysis (for order weights), addressing the method uncertainty problem in multicriteria modelling (Heywood et al. 1994). The interactive review of decision strategy is also an enhancement of previous work on 
OWA done by of Eastman (1997) and Jiang and Eastman (2000) who designed and implemented an OWA module for the Idrisi GIS.

An additional, technical, difference to Eastman's OWA implementation in Idrisi is the application to vector-based spatial data. This implies combinations of columns in a feature attribute table instead of combinations of criterion maps through map algebra. A potential benefit is that OWA could be made accessible to a range of standard database applications. Database queries would use OWA to evaluate search criteria and establish a ranking of search results. In return, GIS that are connected to standard databases would not have to provide their own OWA multicriteria models.

The OWA implementation in CommonGIS extends the work of Jankowski et al. (2001) on multicriteria decision analysis in an exploratory, Webenabled framework and thus addresses the suggestion by Heywood et al. (1994) to provide more than one evaluation method in the same system to allow for comparison of evaluation results. The options for comparison in CommonGIS include interactive scatter plots of evaluation results as shown in Section 4. The sample application also proved that CommonGIS is a useful SDSS generator (as defined by Keenan 1997).

Our future research will be directed towards empirical studies of spatial OWA applications in real-world problems in order to evaluate and improve the usability of the method itself as well as its presentation in the software. Also, an empirical study should be undertaken to provide insight into the efficiency and effectiveness of spatial decision-making processes that make use of an exploratory OWA implementation in GIS. For example, we anticipate that the usability of OWA can be increased through the use of linguistic (fuzzy) quantifiers to define decision strategies (Yager 1988).

Another research task addresses innovative architectures for spatial decision support, and interoperability issues. In the context of OpenGIS, spatial multicriteria evaluation could be made available as services rather than systems. Consequently, OWA decision services on the Web need to be designed and analysed.

\section{References}

Andrienko G, Andrienko N (1999) Interactive maps for visual data exploration. International Journal Geographical Information Science 13(4):355-374

Andrienko G, Andrienko N (2002) Interactive visual tools for spatial multicriteria decision making. Proceedings of ACM Advanced Visual Interfaces ( AVI'02), May 22-24, Trento, Italy, pp. 129-132

Bhargava HK, Tettelbach CG (1997) A Web-based DSS for waste disposal and recycling. Computers, Environment and Urban Systems 21(1):47-65

Banai R (1993) Fuzziness in geographic information systems: Contributions from the analytic hierarchy process. International Journal of Geographical Information Systems 7(4):315-329

Bodily SE (1985) Modern decision making: A guide to modeling with decision support systems. McGraw-Hill, New York

Carver SJ (1991) Integrating multi-criteria evaluation with geographical information systems. International Journal of Geographical Information Systems 5(3):321-339

Carver SJ, Blake M, Turton I (1996) Open spatial decision making: Where to dispose of Britain's radioactive waste? http://www.ccg.leeds.ac.uk/mce [accessed: 07 Jan 2003] 
Carver SJ (1999) Developing Web-based GIS/MCE: Improving access to data and spatial decision support tools. In: Thill J-C (ed) Multicriteria decision-making and analysis: A geographic information sciences approach. Ashgate, New York, pp. 49-76

CommonGIS (2002a) Demos of CommonGIS prototype, http://commongis.jrc.it/demos.html [accessed: 29 Dec 2002]

CommonGIS (2002b) CommonGIS distribution download page, http://www.commongis.com/ download.html [accessed: 29 Dec 2002]

Eastman JR (1997) IDRISI for Windows, Version 2.0: Tutorial exercises. Graduate School of Geography, Clark University, Worcester [MA]

Fraunhofer (2002) Knowledge Discovery Team, http://ais.gmd.de/KD [accessed: 29 Dec 2002]

Heywood I, Oliver J, Tomlinson S (1994) Building an exploratory multi criteria modelling environment for spatial decision support. Proceedings of the 5th European Conference and Exhibition on Geographical Information Systems (EGIS), March 29 - April 01, Paris, pp. 632641

Jankowski P (1995) Integrating geographical information systems and multiple criteria decision making methods. International Journal of Geographical Information Systems 9(3):251-273

Jankowski P, Andrienko N, Andrienko G (2001) Map-centered exploratory approach to multiple criteria spatial decision making. International Journal of Geographical Information Science 15(2):101-127

Janssen R, Rietveld P (1990) Multicriteria analysis and geographical information systems: An application to agricultural land use in the Netherlands. In: Scholten HJ, Stillwell JCH (eds) Geographical information systems for urban and regional planning. Kluwer Academic Publishers, Dordrecht, pp. 129-139

Jiang H, Eastman JR (2000) Application of fuzzy measures in multi-criteria evaluation in GIS. International Journal of Geographical Information Systems 14(2):173-184

Joerin F, Thériault M, Musy A (2001) Using GIS and outranking multicriteria analysis for landuse suitability assessment. International Journal of Geographical Information Science 15(2):153-174

Keenan P (1997) Using a GIS as a DSS generator. Working Paper MIS 95-9, University College Dublin, Ireland. Available at http://mis.ucd.ie/staff/pkeenan/gis_as_a_dss.html [accessed 18 Sept 2002]

Malczewski J (1996) A GIS-based approach to multiple criteria group decision-making. International Journal of Geographical Information Science 10(8):955-971

Malczewski J (1999) GIS and multicriteria decision analysis. John Wiley, New York

Pereira JMC, Duckstein L (1993) A multiple criteria decision-making approach to GIS-based land suitability evaluation. International Journal of Geographical Information Systems 7(5):407-424

MapQuest (2002) MapQuest.com, http://www.mapquest.com [accessed 29 Dec 2002]

Menegolo L, Peckham RJ (1996) A fully integrated tool for site planning using multi criteria evaluation techniques within a GIS. In: Rumor M, McMillan R, Ottens HFL (eds) Geographical information. IOSA Press, Amsterdam, pp. 621-630

MLS (2002) Multiple listing service, http://www.mls.ca [accessed 29 Dec 2002]

OWA (2002) OWA in CommonGIS demo application, http://ifgi.uni-muenster.de/ rinner/ papers/jgs2002 [accessed 07 Jan 2003]

Thill J-C (1999) Multicriteria decision-making and analysis: A geographic information sciences Approach. Ashgate, New York

Voß H, Andrienko N, Andrienko G (2000) CommonGIS - Common access to geographically referenced data. ERCIM News 41:44-46. (Available online at http://www.ercim.org/publication/Ercim_News/enw41/voss.html [accessed 09 Sept 2002])

Voß A, Voß $\bar{H}$, Gatalsky P, Oppor L (2002) Group decision support for spatial planning. Paper submitted to the Urban Data Management Symposium, October 01-04, Prague. Available online at http://www.ais.fraunhofer.de/MS/pdf/UDMS-2002-Voss.pdf [accessed 07 Jan 2003]

Wallis (2002) CommonGIS: Skiing resorts in Wallis, http://borneo.gmd.de/and/CommonGISApplet/wallis.html [accessed 29 Dec 2002] 
Wan Q, Zhang J, Lin H (1999) On-line group spatial decision support system for investment environment analysis. Proceedings of Geoinformatics'99 Conference, June 19-21, Ann Arbor, pp. 1-8

Yager RR (1988) On ordered weighted averaging aggregation operators in multi-criteria decision making. IEEE Transactions on Systems, Man and Cybernetics 18(1):183-190

Yager RR (1997) On the inclusion of importances in OWA aggregations. In: Yager RR, Kacprzyk J (eds) The ordered weighted averaging operators: Theory and applications. Kluwer Academic Publisher, Boston, pp. 41-59

Zhu X, Dale AP (2001) JavaAHP: A Web-based decision analysis tool for natural resource and environmental management. Environmental Modelling \& Software 16(3):251-262

Zhu X, McCosker J, Dale AP, Bischof RJ (2001) Web-based decision support for regional vegetation management. Computers, Environment and Urban Systems 25(6):605-627 